\title{
A Special Class of Univalent Functions in Hele-Shaw Flow Problems
}

\author{
Paula Curt ${ }^{1}$ and Denisa Fericean ${ }^{2}$ \\ ${ }^{1}$ Faculty of Economics and Business Administration, Babeş-Bolyai University, \\ 400591 Cluj-Napoca, Romania \\ ${ }^{2}$ Faculty of Mathematics and Computer Science, Babeş-Bolyai University, \\ 400084 Cluj-Napoca, Romania
}

Correspondence should be addressed to Paula Curt, paula.curt@econ.ubbcluj.ro

Received 7 December 2010; Accepted 23 February 2011

Academic Editor: Yoshikazu Giga

Copyright (C) 2011 P. Curt and D. Fericean. This is an open access article distributed under the Creative Commons Attribution License, which permits unrestricted use, distribution, and reproduction in any medium, provided the original work is properly cited.

We study the time evolution of the free boundary of a viscous fluid for planar flows in HeleShaw cells under injection. Applying methods from the theory of univalent functions, we prove the invariance in time of $\Phi$-likeness property (a geometric property which includes starlikeness and spiral-likeness) for two basic cases: the inner problem and the outer problem. We study both zero and nonzero surface tension models. Certain particular cases are also presented.

\section{Introduction}

The time evolution of the free boundary of a viscous fluid for planar flows in Hele-Shaw cells under injection was studied by many authors. By using methods of univalent functions theory, they proved that certain geometric properties (such as starlikeness and directional convexity) are preserved in time [1-6]. In this paper, we continue their study by proving the invariance in time of another geometric property: $\Phi$-likeness.

In the first section of the paper, we review the main results that are needed later. We start by presenting the basic notions regarding the bounded case.

In this case, we study the flow of a viscous fluid in a planar Hele-Shaw cell under injection through a source (of constant strength $Q, Q<0$ in case of injection) which is situated at the origin. Suppose that at the initial moment the domain $\Omega(0)$ occupied by the fluid is simply connected and is bounded by an analytic and smooth curve $\Gamma(0)=\partial \Omega(0)$. By using the well-known Riemann mapping theorem, the domain $\Omega(t)$ (occupied by the fluid at the moment $t$ ) can be described by a univalent function $f(\zeta, t)$ of the unit disk $U=\{\zeta:|\zeta|<1\}$ 
onto $\Omega(t)$ normalized by $f(0, t)=0, f^{\prime}(0, t)>0$. We denote $\Gamma(t)=\partial \Omega(t)$. The function $f(\zeta, 0)=$ $f_{0}(\zeta)$ produces a parametrization of $\Gamma_{0}=\left\{f_{0}\left(e^{i \theta}\right), \theta \in[0,2 \pi)\right\}$, and the moving boundary is parameterized by $\Gamma(t)=\left\{f\left(e^{i \theta}, t\right), \theta \in[0,2 \pi)\right\}$.

The equation satisfied by the free boundary was first derived by Galin [7] and Polubarinova-Kochina [8, 9]:

$$
\operatorname{Re}\left[\dot{f}(\zeta, t) \overline{\zeta f^{\prime}(\zeta, t)}\right]=-\frac{Q}{2 \pi}, \quad \zeta=e^{i \theta}
$$

(in the previous equality we have used the notations: $f^{\prime}=\partial f / \partial \zeta, \dot{f}=\partial f / \partial t$ ).

A classical solution in the interval $[0, T)$ is a function $f(\zeta, t), t \in[0, T)$, that is univalent on $\bar{U}$ and $C^{1}$ with respect to $t$ in $[0, T)$. It is known that, starting with an analytic and smooth boundary $\Gamma(0)$, the classical solution exists and is unique locally in time (see [10]; see e.g., [11, Chapter 1]). Note that $T$ is called the blow-up time.

In the case of the problem of injection $(Q<0)$ of the fluid into a bounded simply connected with small surface tension $\gamma>0$, the Polubarinova-Galin equation [6] is of the form:

$$
\operatorname{Re}\left[\dot{f}(\zeta, t) \overline{\zeta f^{\prime}(\zeta, t)}\right]=-\frac{Q}{2 \pi}+\gamma H\left[i \frac{\partial k}{\partial \theta}\left(e^{i \theta}, t\right)\right](\theta), \quad \zeta=e^{i \theta}
$$

where $k\left(e^{i \theta}, t\right)=\left(1 /\left|f^{\prime}\left(e^{i \theta}, t\right)\right|\right) \operatorname{Re}\left(1+\left(e^{i \theta} f^{\prime \prime}\left(e^{i \theta}, t\right) / f^{\prime}\left(e^{i \theta}, t\right)\right)\right), \theta \in[0,2 \pi)$, and the Hilbert transform in (1.2) is given by $(1 / \pi)$ P.V. $\theta \int_{0}^{2 \pi} \Phi\left(e^{i \theta^{\prime}}\right) /\left(1-e^{i\left(\theta-\theta^{\prime}\right)}\right) d \theta^{\prime}=H[\Phi](\theta)$.

We mention the following technical results: $(\partial k / \partial \theta)\left(e^{i \theta}, t\right)=$ $-\operatorname{Im}\left(e^{2 i \theta} S_{f}\left(e^{i \theta}, t\right)\right) /\left|f^{\prime}\left(e^{i \theta}, t\right)\right|$ where $S_{f}$ is the Schwarzian derivative given by $S_{f}(\zeta)=\left(f^{\prime \prime}(\zeta, t) / f^{\prime}(\zeta, t)\right)^{\prime}-(1 / 2)\left(f^{\prime \prime}(\zeta, t) / f^{\prime}(\zeta, t)\right)^{2}$ and

$$
\frac{\partial}{\partial \theta}\left[i \frac{\partial k}{\partial \theta}\left(e^{i \theta}, t\right)\right](\theta)=-\frac{\partial}{\partial \theta}\left[\frac{i \operatorname{Im}\left(e^{2 i \theta} S_{f}\left(e^{i \theta}\right)\right)}{\left|f^{\prime}\left(e^{i \theta}\right)\right|}\right]=H[i A](\theta),
$$

where

$$
\begin{aligned}
A(\zeta)=\left(\frac{1}{\left|f^{\prime}(\zeta)\right|}\right)[ & \operatorname{Re}\left(2 \zeta^{2} S_{f}(\zeta)+\zeta\left[\left(\frac{f^{\prime \prime}(\zeta)}{f^{\prime}(\zeta)}\right)^{\prime}-\frac{f^{\prime \prime}(\zeta)}{f^{\prime}(\zeta)}\left(\frac{f^{\prime \prime}(\zeta)}{f^{\prime}(\zeta)}\right)^{\prime}\right]\right) \\
& \left.+\operatorname{Im} \frac{\zeta f^{\prime \prime}(\zeta)}{f^{\prime}(\zeta)} \operatorname{Im} \zeta^{2} S_{f}(\zeta)\right] .
\end{aligned}
$$

The case of unbounded domain with bounded complement can be viewed as the dynamics of a contracting bubble in a Hele-Shaw cell since the fluid occupies a neighbourhood of infinity and injection (of constant strength $Q<0$ ) is supposed to take place at infinity. Again, we denote by $\Omega(t)$ the domain occupied by the fluid at the moment $t, \Gamma(t)=\partial \Omega(t)$. By using the Riemann mapping theorem, the domain $\Omega(t)$ can be described by a univalent function $F(\zeta, t)$ from the exterior of the unit disk $U^{-}=\{\zeta|| \zeta \mid>1\}$ onto $\Omega(t)$, $F(\zeta, t)=a \zeta+a_{0}+a_{1} / \zeta+\cdots, a>0$. 
The equation satisfied by the free boundary is $[4,6]$

$$
\operatorname{Re}\left[\dot{F}(\zeta, t) \overline{\zeta F^{\prime}(\zeta, t)}\right]=\frac{Q}{2 \pi}, \quad \zeta=e^{i \theta}
$$

for the zero tension surface model and

$$
\operatorname{Re}\left[\dot{F}(\zeta, t) \overline{\zeta F^{\prime}(\zeta, t)}\right]=\frac{Q}{2 \pi}-\gamma H\left[i \frac{\partial k}{\partial t}\left(e^{i \theta}, t\right)\right](\theta), \quad \zeta=e^{i \theta}
$$

for the small surface tension model.

\section{The Inner Problem (Bounded Domains)}

In this section, we obtain the invariance in time of $\Phi$-likeness property for the inner problem. Starting with an initial bounded domain $\Omega(0)$ which is $\Phi$-like, we prove that at each moment $t \in[0, T)$ the domain $\Omega(t)$ is $\Phi$-like (both for zero and nonzero surface tension models).

Definition 2.1. Let $f$ be a holomorphic function on $U$ such that $f(0)=0$ and $f^{\prime}(0) \neq 0$. Let $\Phi$ be a holomorphic function on $f(U)$ such that $\Phi(0)=0$ and $\operatorname{Re} \Phi^{\prime}(0)>0$. We say that $f$ is $\Phi$-like on $U$ (or $\Phi$-like) if

$$
\operatorname{Re}\left[\frac{z f^{\prime}(z)}{\Phi(f(z))}\right]>0, \quad \text { for each } z \in U
$$

We remark that a $\Phi$-like function is univalent. In fact, any univalent function is $\Phi$-like for some $\Phi$.

Remark 2.2. (a) The concept of $\Phi$-likeness was introduced and studied by Brickman in 1973 [12] and generalizes the notions of starlikeness and spiral-likeness. Applications of this notion in the study of univalence may be found in [13].

(b) If $\Phi(w)=w$ in the above definition, then $f$ is starlike.

(c) If $\Phi(w)=\lambda w$ and $\operatorname{Re} \lambda>0$, then $f$ is spiral-like of type $-\arg \lambda$.

We restate that a holomorphic function $f$ on $U$ such that $f(0)=0$ and $f^{\prime}(0) \neq 0$ is said to be spiral-like of type $\alpha \in(-\pi / 2, \pi / 2)$ if $\operatorname{Re}\left(e^{i \alpha} z f^{\prime}(z) / f(z)\right)>0, z \in U[13,14]$.

The following result is a generalization of [1, Theorem 1] to the case of $\Phi$-like functions. The mentioned theorem may be obtained by taking $\Phi(w) \equiv w$ in Theorem 2.3 below.

Theorem 2.3. Let $Q<0$ and $f_{0}$ be a function which is $\Phi$-like on $U$ and univalent on $\bar{U}$. Let $f(\zeta, t)$ be the classical solution of the Polubarinova-Galin equation (1.1) with the initial condition $f(\zeta, 0)=$ $f_{0}(\zeta)$. Also let $\Omega=\bigcup_{0 \leq t<T} \Omega(t)=\bigcup_{0 \leq t<T} f(U, t)$, where $T$ is the blow-up time. If $\Phi$ is holomorphic on $\bar{\Omega}$ and satisfies the condition

$$
\operatorname{Re} \Phi^{\prime}(w)>0, \quad \forall w \in \bar{\Omega}
$$

then $f(\zeta, t)$ is $\Phi$ like for $t \in[0, T)$. 
Proof. Taking into account that all the functions $f(\zeta, t)$ have analytic univalent extensions to $\bar{U}$ for each $t \in[0, T)$ and in consequence their derivatives $f^{\prime}(\zeta, t)$ are continuous and do not vanish in $\bar{U}$, we can replace with " $\geq$ " the inequality in the definition (2.1) of a $\Phi$-like function. The equality can be attained only for $|\zeta|=1$.

We suppose by contrary that the conclusion of Theorem 2.3 is not true. Then there exist $t_{0} \geq 0$ and $\zeta_{0}=e^{i \theta_{0}}$ such that $\arg \left(\zeta_{0} f^{\prime}\left(\zeta_{0}, t_{0}\right) / \Phi\left(f\left(\zeta_{0}, t_{0}\right)\right)\right)=\pi / 2$ (or $\left.-\pi / 2\right)$, which is equivalent to

$$
\operatorname{Re} \frac{\zeta_{0} f^{\prime}\left(\zeta_{0}, t_{0}\right)}{\Phi\left(f\left(\zeta_{0}, t_{0}\right)\right)}=0, \quad \operatorname{Im} \frac{\zeta_{0} f^{\prime}\left(\zeta_{0}, t_{0}\right)}{\Phi\left(f\left(\zeta_{0}, t_{0}\right)\right)} \neq 0
$$

and for each $\varepsilon>0$, there are $t>t_{0}$ and $\theta \in\left(\theta_{0}-\varepsilon, \theta_{0}+\varepsilon\right)$ such that

$$
\arg \frac{e^{i \theta} f^{\prime}\left(e^{i \theta}, t\right)}{\Phi\left(f\left(e^{i \theta}, t\right)\right)} \geq \frac{\pi}{2} \quad\left(\text { or } \leq-\frac{\pi}{2}\right)
$$

Let $t_{0}$ be the first such point, $t_{0} \in[0, T)$. Without loss of generality, we assume that

$$
\operatorname{Im} \frac{\zeta_{0} f^{\prime}\left(\zeta_{0}, t_{0}\right)}{\Phi\left(f\left(\zeta_{0}, t_{0}\right)\right)}>0
$$

In fact, $\left(1, \theta_{0}\right)$ is a maximum point for the function $g(r, \theta)=$ $\arg \left(r e^{i \theta} f^{\prime}\left(r e^{i \theta}, t_{0}\right) / \Phi\left(f\left(r e^{i \theta}, t_{0}\right)\right)\right)$, where $r \in[0,1], \theta \in[0,2 \pi]$. Hence, $(\partial / \partial \theta) g\left(1, \theta_{0}\right)=0$ and $(\partial / \partial r) g\left(1, \theta_{0}\right) \geq 0$ (the stationarity condition at an endpoint of an interval), and in consequence we obtain

$$
\begin{aligned}
& \operatorname{Re}\left(1+\frac{\zeta_{0} f^{\prime \prime}\left(\zeta_{0}, t_{0}\right)}{f^{\prime}\left(\zeta_{0}, t_{0}\right)}-\frac{\Phi^{\prime}\left(f\left(\zeta_{0}, t_{0}\right)\right) \zeta_{0} f^{\prime}\left(\zeta_{0}, t_{0}\right)}{\Phi\left(f\left(\zeta_{0}, t_{0}\right)\right)}\right)=0 \\
& \operatorname{Im}\left(1+\frac{\zeta_{0} f^{\prime \prime}\left(\zeta_{0}, t_{0}\right)}{f^{\prime}\left(\zeta_{0}, t_{0}\right)}-\frac{\Phi^{\prime}\left(f\left(\zeta_{0}, t_{0}\right)\right) \zeta_{0} f^{\prime}\left(\zeta_{0}, t_{0}\right)}{\Phi\left(f\left(\zeta_{0}, t_{0}\right)\right)}\right) \geq 0 .
\end{aligned}
$$

By straightforward calculations, we get

$$
\frac{\partial}{\partial t} \arg \frac{\zeta f^{\prime}(\zeta, t)}{\Phi(f(\zeta, t))}=\operatorname{Im}\left(\frac{(\partial / \partial t) f^{\prime}(\zeta, t)}{f^{\prime}(\zeta, t)}-\frac{\Phi^{\prime}(f(\zeta, t))(\partial / \partial t) f(\zeta, t)}{\Phi(f(\zeta, t))}\right)
$$


By differentiating the Polubarinova-Galin equation with respect to $\theta$, we obtain $\operatorname{Im}\left((\partial / \partial t) f^{\prime}(\zeta, t) \overline{f^{\prime}(\zeta, t)}-\overline{\zeta f^{\prime}(\zeta, t)}(\partial / \partial t) f(\zeta, t)-\overline{\zeta^{2} f^{\prime \prime}(\zeta, t)}(\partial / \partial t) f(\zeta, t)\right)=0$ for $\zeta=e^{i \theta}$. The previous equality yields the following relation:

$$
\begin{aligned}
& \left|f^{\prime}(\zeta, t)\right|^{2} \operatorname{Im}\left(\frac{(\partial / \partial t) f^{\prime}(\zeta, t)}{f^{\prime}(\zeta, t)}-\frac{\Phi^{\prime}(f(\zeta, t)) \cdot(\partial / \partial t) f(\zeta, t)}{\Phi(f(\zeta, t))}\right) \\
& \quad=\operatorname{Im}\left(\overline{\zeta f^{\prime}(\zeta, t)} \dot{f}(\zeta, t)\right)\left(1+\overline{\left(\frac{\zeta f^{\prime \prime}(\zeta, t)}{f^{\prime}(\zeta, t)}\right)}-\frac{\Phi^{\prime}(f(\zeta, t)) \cdot \zeta f^{\prime}(\zeta, t)}{\Phi(f(\zeta, t))}\right) .
\end{aligned}
$$

If we substitute (2.6), (2.7), (2.8), and (1.1) in the above expression and replace $\theta$ by $\theta_{0}$ and $t$ by $t_{0}$, we obtain

$$
\begin{aligned}
\left.\frac{\partial}{\partial t} \arg \frac{\zeta f^{\prime}(\zeta, t)}{\Phi(f(\zeta, t))}\right|_{\zeta=\zeta_{0}, t=t_{0}}=\frac{Q}{2 \pi\left|f^{\prime}\left(\zeta_{0}, t_{0}\right)\right|^{2}} & {\left[\operatorname{Im}\left(\frac{\zeta_{0} f^{\prime \prime}\left(\zeta_{0}, t_{0}\right)}{f^{\prime}\left(\zeta_{0}, t_{0}\right)}-\frac{\Phi^{\prime}\left(f\left(\zeta_{0}, t_{0}\right) \zeta_{0} f^{\prime}\left(\zeta_{0}, t_{0}\right)\right)}{\Phi\left(f\left(\zeta_{0}, t_{0}\right)\right)}\right)\right.} \\
& \left.+2 \operatorname{Re} \Phi^{\prime}\left(f\left(\zeta_{0}, t_{0}\right)\right) \operatorname{Im} \frac{\zeta_{0} f^{\prime}\left(\zeta_{0}, t_{0}\right)}{\Phi\left(f\left(\zeta_{0}, t_{0}\right)\right)}\right]<0,
\end{aligned}
$$

due to (2.2), (2.3) and (2.5). Finally, we get $\left.(\partial / \partial t) \arg \left(\zeta f^{\prime}(\zeta, t) / \Phi(f(\zeta, t))\right)\right|_{\zeta=\zeta_{0}, t=t_{0}}<0$. Therefore, $\arg \left(e^{i \theta} f^{\prime}\left(e^{i \theta}, t\right) / \Phi\left(f\left(e^{i \theta}, t\right)\right)\right)<\pi / 2$, for $t>t_{0}$ (close to $t_{0}$ ) in some neighbourhood of $\theta_{0}$. This contradicts the assumption (2.4) and completes the proof.

If in the previous theorem, we take $\Phi(w) \equiv e^{-i \alpha} w, \alpha \in(-\pi / 2, \pi / 2)$; then we obtain the following corollary.

Corollary 2.4. Let $Q<0$, and let $f_{0}$ be a function which is spiral-like of type $\alpha \in(-\pi / 2, \pi / 2)$ on $U$ and univalent on $\bar{U}$. Then the classical solution of the Polubarinova-Galin equation (1.1) with the initial condition $f(\zeta, 0)=f_{0}(\zeta)$ is spiral-like of type a for $t \in[0, T)$, where $T$ is the blow-up time.

The following result is a generalization of [6, Theorem 1] to the case of $\Phi$-like functions. The mentioned theorem may be obtained by taking $\Phi(w) \equiv w$ in Theorem 2.5 below.

Theorem 2.5. Let $Q<0$ and the surface tension $\gamma$ be sufficiently small. If $f_{0}$ is a function which is $\Phi$-like on $U$ and univalent on $\bar{U}$, then there exists $t(\gamma) \leq T$ such that the classical solution $f(\zeta, t)$ of (1.2) with the initial condition $f(\zeta, 0)=f_{0}(\zeta)$ is $\Phi$-like for $t \in[0, t(\gamma)$ ), where $T$ is the blow-up time, $\Omega=\bigcup_{0 \leq t<t(\gamma)} \Omega(t)=\bigcup_{0 \leq t<t(\gamma)} f(U, t)$, and $\Phi$ is a holomorphic function on $\bar{\Omega}$ which satisfies the condition (2.2).

Proof. If we consider $f$ in the closure of $U$, then the inequality sign in (2.1) can be replaced by " $\geq$ " where equality can be attained for $|\zeta|=1$.

Suppose by contrary that the conclusion of Theorem 2.5 is not true. Then there exist $t_{0} \geq 0$ and $\zeta_{0}=e^{i \theta_{0}}$ such that (2.3), (2.4), (2.5), (2.6), and (2.7) are true. At the same time 
the equality (2.8) is fulfilled. By differentiating the Polubarinova-Galin equation (1.2) with respect to $\theta$, we obtain

$$
\operatorname{Im}\left(\frac{\partial}{\partial t} f^{\prime}(\zeta, t) \overline{f^{\prime}(\zeta, t)}-\overline{\zeta f^{\prime}(\zeta, t)} \frac{\partial}{\partial t} f(\zeta, t)-\overline{\zeta^{2} f^{\prime \prime}(\zeta, t)} \frac{\partial}{\partial t} f(\zeta, t)\right)=\gamma H[i A](\theta),
$$

for $\zeta=e^{i \theta}$. The previous equality is equivalent to the following:

$$
\begin{aligned}
\left.\left|f^{\prime}(\zeta, t)\right|^{2} \operatorname{Im}\left(\frac{(\partial / \partial t) f^{\prime}(\zeta, t)}{f^{\prime}(\zeta, t)}-\frac{\Phi(f(\zeta, t))(\partial / \partial t) f(\zeta, t)}{\Phi(f(\zeta, t))}\right)\right|_{\zeta=\zeta_{0}=e^{i \theta_{0}, t=t_{0}}} \\
=\left(\frac{Q}{2 \pi}-\gamma H\left(i \frac{\partial k}{\partial \theta}\left(e^{i \theta}, t\right)\right)\right) \\
\quad \times\left.\left[\operatorname{Im}\left(\frac{\zeta f^{\prime \prime}}{f^{\prime}}-\frac{\Phi^{\prime}(f)}{\Phi(f)} \zeta f^{\prime}\right)+2 \operatorname{Re} \Phi^{\prime}(f) \operatorname{Im} \frac{\zeta f^{\prime}}{\Phi(f)}\right]\right|_{\zeta=\zeta_{0}, t=t_{0}, \theta=\theta_{0}}-\left.\gamma H[i A](\theta)\right|_{\zeta=\zeta_{0}, t=t_{0}, \theta=\theta_{0}} .
\end{aligned}
$$

The right-hand side of this equality is strictly negative for small $\gamma$ because of (2.5), (2.7), and the fact that $\operatorname{Re} \Phi^{\prime}(f)>0$.

By using (2.8), we obtain that $\arg \left(e^{i \theta} f^{\prime}\left(e^{i \theta}, t\right) / \Phi\left(f\left(e^{i \theta}, t\right)\right)\right)<\pi / 2$ for $t>t_{0}$ (close to $\left.t_{0}\right)$ in a neighbourhood of $\theta_{0}$. This is in contradiction with our assumption and ends the proof.

Remark 2.6. Let $Q<0$, and let $f_{0}$ be a function that is $\Phi$-like on $U$ and univalent on $\bar{U}$. If $f_{0}$ satisfies the condition (2.1) for each $\zeta \in \bar{U}$, then there exist a surface tension $\gamma$ (which depends on $f_{0}$ ) sufficiently small and $t(\gamma) \leq T$ such that the classical solution $f(\zeta, t)$ of (1.2) with the initial condition $f(\zeta, 0)=f_{0}(\zeta)$ are $\Phi$-like for $t \in[0, t(\gamma))$, where $T$ is the blow-up time.

Proof. The conclusion is immediate from the smoothness of the classical solution of (1.1). corollary:

If $\Phi(w) \equiv e^{-i \alpha} w$ in Theorem 2.5, where $\alpha \in(-\pi / 2, \pi / 2)$, then we obtain the following

Corollary 2.7. Let $Q<0$ and the surface tension $\gamma$ be sufficiently small. If $f_{0}$ is a function which is spiral-like of type $\alpha \in(-\pi / 2, \pi / 2)$ on $U$ and univalent on $\bar{U}$, then there exists $t(\gamma) \leq T$ such that the classical solution $f(\zeta, t)$ of (1.2) with the initial condition $f(\zeta, 0)=f_{0}(\zeta)$ is spiral-like of type $\alpha$ for $t \in[0, t(\gamma))$, where $T$ is the blow-up time.

\section{The Outer Problem (Unbounded Domain with Bounded Complement)}

In this section, we obtain the invariance in time of the same geometric property (denoted by $\widetilde{\Phi})$ for the outer problem. 
Definition 3.1. Let $F$ be a holomorphic function on $U^{-}=\{\zeta|| \zeta \mid>1\}$ such that $F(\zeta)=a \zeta+a_{0}+$ $a_{-1} / \zeta+\cdots$, where $a \neq 0$. Let $\widetilde{\Phi}$ be a holomorphic function on $F\left(U^{-}\right)$such that $\lim _{\zeta \rightarrow \infty} \widetilde{\Phi}(\zeta)=\infty$ and $\lim _{\zeta \rightarrow \infty} \widetilde{\Phi}^{\prime}(\zeta)>0$. We say that $F$ is $\widetilde{\Phi}$-like on $U^{-}$if

$$
\operatorname{Re}\left[\frac{\zeta F^{\prime}(\zeta)}{\widetilde{\Phi}(F(\zeta))}\right]>0, \quad \zeta \in U^{-}
$$

Remark 3.2. (a) If $F$ is a $\widetilde{\Phi}$-like function on $U^{-}$, then the function $f: U \rightarrow \mathrm{C}$ given by $f(z)=$ $1 / F(1 / z), z \neq 0$, and $f(0)=0$, is $\Phi$-like on $U$, where $\Phi: f(U) \rightarrow \mathbf{C}, \Phi(w)=w^{2} \widetilde{\Phi}(1 / w)$, for all $w \in f(U) \backslash\{0\}$ and $\Phi(0)=0$.

(b) If $f$ is a $\Phi$-like function on $U$, then the function $F: U^{-} \rightarrow \mathbf{C}, F(\zeta)=1 / f(1 / \zeta)$ is $\widetilde{\Phi}$-like on $U^{-}$, where $\widetilde{\Phi}: F\left(U^{-}\right) \rightarrow \mathrm{C}, \widetilde{\Phi}(\omega)=\omega^{2} \Phi(1 / \omega)$, for all $\omega \in F\left(U^{-}\right)$.

(c) Any $\widetilde{\Phi}$-like holomorphic function $F$ on $U^{-}$is univalent on $U^{-}$.

Proof. This remark can be obtained by straightforward computations and its proof is, therefore, left to the reader.

The following result is a generalization of [5, Theorem 3]. The mentioned theorem may be obtained by taking $\widetilde{\Phi}(w) \equiv w$ in Theorem 3.3 below.

Theorem 3.3. Let $F_{0}$ be a function which is $\widetilde{\Phi}$-like on $U^{-}$and univalent on $\overline{U^{-}}$. Then the solution $F(\zeta, t)$ of the Polubarinova-Galin equation (1.5) with the initial condition $F(\zeta, 0)=F_{0}(\zeta)$ is $\widetilde{\Phi}$-like for $t \in[0, T)$, where $T$ is the blow-up time, $\Omega=\bigcup_{0 \leq t<T} \Omega(t)=\bigcup_{0 \leq t<T} F\left(U^{-}, t\right)$, and $\widetilde{\Phi}$ is a holomorphic function on $\bar{\Omega}$ which satisfies the following conditions:

$$
\operatorname{Re} \frac{\widetilde{\Phi}(\omega)}{\omega}>0, \quad \operatorname{Re} \tilde{\Phi}^{\prime}(\omega)<2 \operatorname{Re} \frac{\widetilde{\Phi}(\omega)}{\omega}, \quad \forall \omega \in \bar{\Omega}
$$

Proof. By considering the function $f(\zeta, t)=1 / F(1 / \zeta, t)$, the Polubarinova-Galin equation (1.5) can be rewritten in terms of $f$ as follows:

$$
\operatorname{Re} \dot{f}(\zeta, t) \overline{\zeta f^{\prime}(\zeta, t)}=-\frac{Q|f(\zeta, t)|^{4}}{2 \pi}, \quad|\zeta|=1
$$

Due to the previous remark, the function $F(\zeta, t), \zeta \in U^{-}$, is $\widetilde{\Phi}$-like if and only if $f(\zeta, t), \zeta \in$ $U$, is $\Phi$-like, where the relationship between $\widetilde{\Phi}$ and $\Phi$ is $\widetilde{\Phi}(\omega)=\omega^{2} \Phi(1 / \omega)$, for all $\omega \in$ $F\left(U^{-}\right)$(or $\Phi(w)=w^{2} \widetilde{\Phi}(1 / w)$, for all $\left.w \in f(U)\right)$. Thus, it suffices to prove that the functions $f(\zeta, t)$ are $\Phi$-like for $t \in[0, T)$. 
Suppose by contrary that the previous statement is not true. Then there exist $t_{0} \geq 0$ and $\zeta_{0}=e^{i \theta_{0}}$ such that (2.3), (2.4), (2.5), (2.6), and (2.7) are true. At the same time, the equality (2.8) is fulfilled. We have to determine the sign of the function $(\partial / \partial t) \arg \left(\zeta f^{\prime}(\zeta, t) / \Phi(f(\zeta, t))\right)$ at the point $\left(\zeta_{0}, t_{0}\right)$.

By differentiating (3.3) and using (2.6), (2.8), and (3.3), we get

$$
\begin{aligned}
& \left.\frac{\partial}{\partial t} \arg \frac{\zeta f^{\prime}(\zeta, t)}{\Phi(f(\zeta, t))}\right|_{\zeta=\zeta_{0}, t=t_{0}} \\
& \quad=\left.\frac{Q|f|^{4}}{2 \pi\left|f^{\prime}\right|^{2}} \operatorname{Im}\left[\left(\frac{\zeta f^{\prime \prime}}{f^{\prime}}-\frac{\Phi^{\prime}(f) \zeta f^{\prime}}{\Phi(f)}\right)+2 \operatorname{Im} \frac{\zeta f^{\prime}}{\Phi(f)}\left(\operatorname{Re} \Phi^{\prime}(f)+2 \operatorname{Re} \frac{\Phi(f)}{f}\right)\right]\right|_{\zeta=\zeta_{0}, t=t_{0}} .
\end{aligned}
$$

The right-hand side of the previous equality is strictly negative in view of (2.5), (2.7), $\operatorname{Re} \Phi^{\prime}(f)>0$, and $\operatorname{Re}(\Phi(f) / f)>0$ (the previous inequalities are easy consequences of (3.2)). Therefore, $\arg \left(e^{i \theta} f^{\prime}\left(e^{i \theta}, t\right) / \Phi\left(f\left(e^{i \theta}, t\right)\right)\right)<\pi / 2$, for $t>t_{0}$ (close to $t_{0}$ ) in some neighbourhood of $\theta_{0}$. This contradiction completes the proof.

The following result is a generalization of [6, Theorem 3.1]. The mentioned theorem may be obtained by taking $\widetilde{\Phi}(w) \equiv w$ in Theorem 3.4 below.

Theorem 3.4. Let $Q<0$, and let the surface tension $\gamma$ be sufficiently small. If $F_{0}$ is a function which is $\widetilde{\Phi}$-like on $U^{-}$and univalent on $\overline{U^{-}}$, then there exists $t(\gamma) \leq T$ such that the solution $F(\zeta, t)$ of $(1.6)$ with the initial condition $F(\zeta, 0)=F_{0}(\zeta)$ is $\widetilde{\Phi}$-like for $t \in[0, t(\gamma))$, where $T$ is the blow-up time, $\Omega=\bigcup_{0 \leq t<t(r)} \Omega(t)=\bigcup_{0 \leq t<t(r)} F\left(U^{-}, t\right)$, and $\widetilde{\Phi}$ is a holomorphic function on $\bar{\Omega}$ which satisfies the conditions (3.2).

Proof. We introduce (as in the proof of Theorem 3.3) the function $f(\zeta, t)=1 / F(1 / \zeta, t), \zeta \in U$, which is $\Phi$-like $\left(\Phi(w)=w^{2} \widetilde{\Phi}(1 / w)\right)$ if and only if $F(\zeta, t)$ is $\widetilde{\Phi}$-like.

The Polubarinova-Galin equation can be written in terms of $f$ as

$$
\operatorname{Re} \dot{f}(\zeta, t) \overline{\zeta f^{\prime}(\zeta, t)}=-|f|^{4}\left(\frac{Q}{2 \pi}-\gamma H\left[i \frac{\partial k}{\partial \theta}\right](\theta)\right), \quad|\zeta|=1
$$

Suppose by contrary that the conclusion of Theorem 3.4 is not true. Then there exist $t_{0} \geq 0$ and $\zeta_{0}=e^{i \theta_{0}}$ such that $(2.3),(2.4),(2.5),(2.6)$, and (2.7) are true. At the same time, the equality (2.8) is fulfilled. We differentiate (3.5) with respect to $\theta$. Since the left side is differentiable with respect to $\theta$ and the solution of (3.5) exists and is unique, then the right-hand side is differentiable and its derivative is bounded on $[0,2 \pi]$. 
If we denote $A(\theta, t)=(\partial / \partial \theta) H[i \partial k / \partial \theta](\theta)$; then by using (2.6), (2.8) and (3.5), we obtain

$$
\begin{aligned}
\frac{\partial}{\partial t} \arg & \left.\frac{\zeta f^{\prime}(\zeta, t)}{\Phi(f(\zeta, t))}\right|_{\zeta=\zeta_{0}=e^{i \theta_{0}, t=t_{0}}} \\
= & \frac{|f|^{4}}{\left|f^{\prime}\right|^{2}}\left(\frac{Q}{2 \pi}-\gamma H\left(i \frac{\partial k}{\partial \theta}\right)(\theta)\right) \\
& \times\left.\left[\operatorname{Im}\left(\frac{\zeta f^{\prime \prime}}{f^{\prime}}-\frac{\Phi^{\prime}(f) \zeta f^{\prime}}{\Phi(f)}\right)+\operatorname{Im} \frac{\zeta f^{\prime}}{\Phi(f)}\left(2 \operatorname{Re} \Phi^{\prime}(f)+4 \operatorname{Re} \frac{\Phi(f)}{f}\right)\right]\right|_{\zeta=\zeta_{0}, t=t_{0}} \\
& +\left.\frac{|f|^{4}}{\left|f^{\prime}\right|^{2}} \cdot \gamma A(\theta, t)\right|_{\zeta=\zeta_{0}, t=t_{0}} .
\end{aligned}
$$

The right-hand side of the previous equality is strictly negative for small $\gamma$ because of (2.5), (2.7), $\operatorname{Re} \Phi^{\prime}(f)>0$, and $\operatorname{Re}(\Phi(f) / f)>0$. Therefore, $\arg \left(e^{i \theta} f^{\prime}\left(e^{i \theta}, t\right) / \Phi\left(f\left(e^{i \theta}, t\right)\right)\right)<$ $\pi / 2$, for $t>t_{0}$ (close to $t_{0}$ ) in some neighbourhood of $\theta_{0}$. This contradiction completes the proof.

Remark 3.5. Let $Q<0$, and let $F_{0}$ be a function that is $\widetilde{\Phi}$-like on $U^{-}$and univalent on $\overline{U^{-}}$. If $F_{0}$ satisfies the condition (3.1) for each $\zeta \in \overline{U^{-}}$, then there exist a surface tension $\gamma$ (which depends on $F_{0}$ ) sufficiently small and $t(\gamma) \leq T$ such that the classical solution $F(\zeta, t)$ of $(1.6)$ with the initial condition $F(\zeta, 0)=F_{0}(\zeta)$ is $\widetilde{\Phi}$-like for $t \in[0, t(\gamma))$, where $T$ is the blow-up time.

Proof. The conclusion is immediate from the smoothness of the classical solution of (1.6)

\section{Acknowledgments}

The authors are indebted to Gabriela Kohr for suggesting this subject and for valuable discussions during the preparation of this paper. Denisa Fericean is supported by Contract no. POSDRU / 88/1.5/S/60185-“Innovative Doctoral Studies in a Knowledge-Based Society". The authors thank the referee for useful suggestions that improved the aspects of the paper.

\section{References}

[1] Y. E. Hohlov, D. V. Prokhorov, and A. J. Vasil'ev, "On geometrical properties of free boundaries in the Hele-Shaw flows moving boundary problem," Lobacherskii Journal of Mathematics, vol. 1, pp. 3-12, 1998.

[2] K. Kornev and A. Vasil'ev, "Geometric properties of the solutions of a Hele-Shaw type equation," Proceedings of the American Mathematical Society, vol. 128, no. 9, pp. 2683-2685, 2000.

[3] D. Prokhorov and A. Vasil'ev, "Convex dynamics in Hele-Shaw cells," International Journal of Mathematics and Mathematical Sciences, vol. 31, no. 11, pp. 639-650, 2002.

[4] A. Vasil'ev, "Univalent functions in the dynamics of viscous flows," Computational Methods and Function Theory, vol. 1, no. 2, pp. 311-337, 2001.

[5] A. Vasil'ev, "Univalent functions in two-dimensional free boundary problems," Acta Applicandae Mathematicae, vol. 79, no. 3, pp. 249-280, 2003. 
[6] A. Vasil'ev and I. Markina, "On the geometry of Hele-Shaw flows with small surface tension," Interfaces and Free Boundaries, vol. 5, no. 2, pp. 183-192, 2003.

[7] L. A. Galin, "Unsteady filtration with a free surface," Doklady Akademii Nauk USSR, vol. 47, pp. 246249, 1945.

[8] P. Y. Polubarinova-Kochina, "On a problem of the motion of the contour of a petroleum shell," Doklady Akademii Nauk USSR, vol. 47, pp. 254-257, 1945 (Russian).

[9] P. J. Poloubarinova-Kochina, "Concerning unsteady motions in the theory of filtration," vol. 9, no. 1, pp. 79-90, 1945 (Russian).

[10] Y. P. Vinogradov and P. P. Kufarev, "On a problem of filtration," Akademii Nauk USSR, vol. 12, pp. 181-198, 1948 (Russian).

[11] B. Gustafsson and A. Vasil'ev, Conformal and Potential Analysis in Hele-Shaw Cells, Advances in Mathematical Fluid Mechanics, Birkhäuser, Basel, Switzerland, 2006.

[12] L. Brickman, "Фَ-like analytic functions-I," Bulletin of the American Mathematical Society, vol. 79, pp. 555-558, 1973.

[13] I. Graham and G. Kohr, Geometric Function Theory in One and Higher Dimensions, vol. 255 of Monographs and Textbooks in Pure and Applied Mathematics, Marcel Dekker, New York, NY, USA, 2003.

[14] C. Pommerenke, Univalent Functions, Vandenhoeck \& Ruprecht, Göttingen, Germany, 1975. 


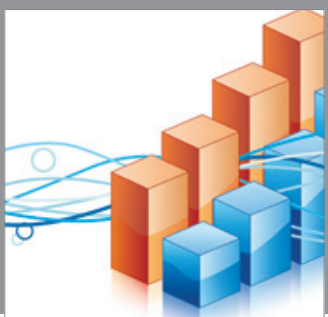

Advances in

Operations Research

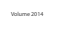

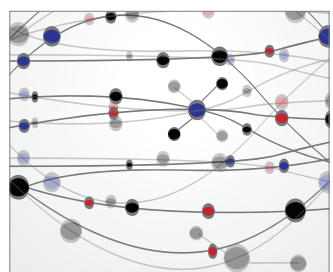

\section{The Scientific} World Journal
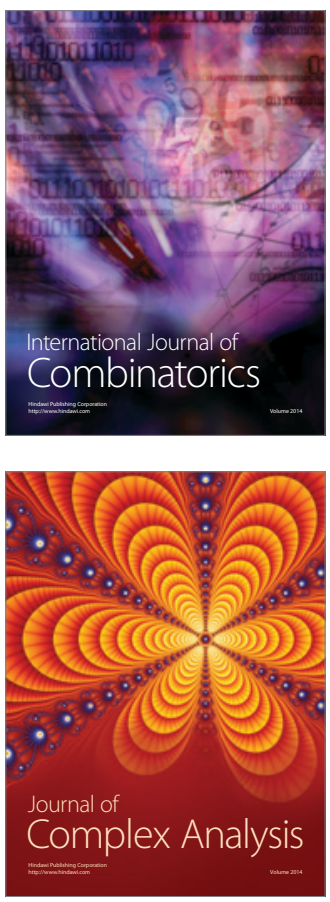

International Journal of

Mathematics and

Mathematical

Sciences
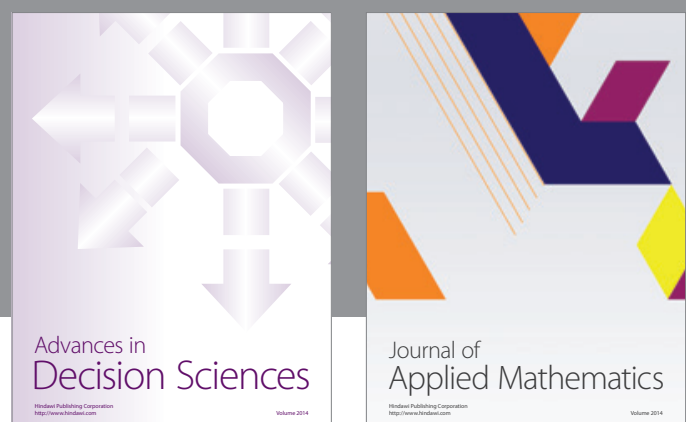

Journal of

Applied Mathematics
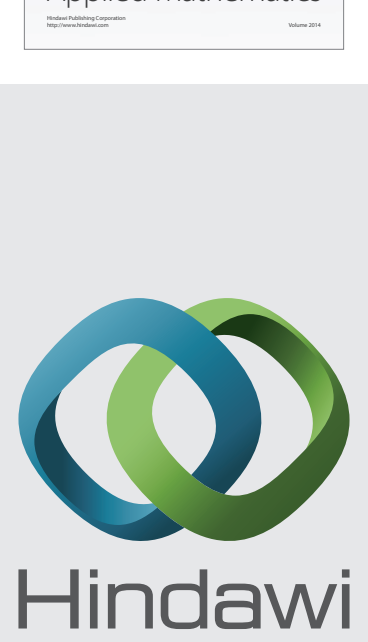

Submit your manuscripts at http://www.hindawi.com
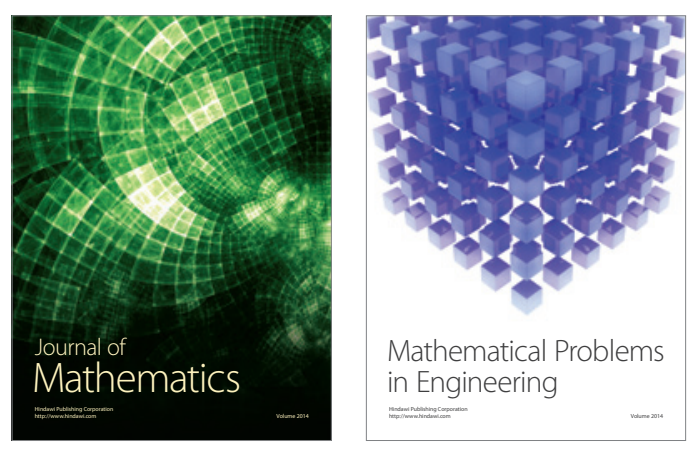

Mathematical Problems in Engineering
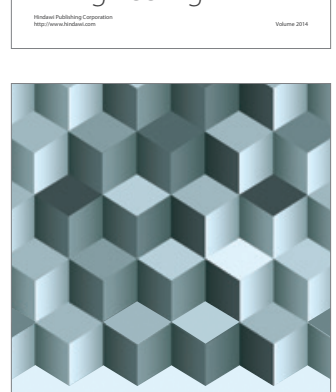

Journal of

Function Spaces
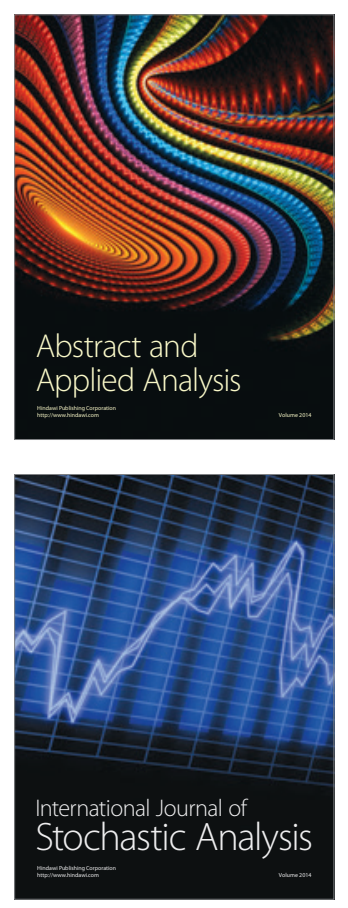

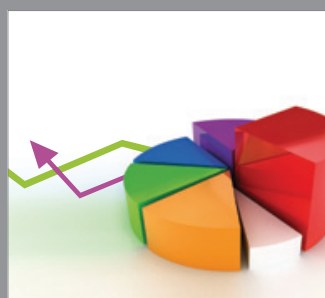

ournal of

Probability and Statistics

Promensencen
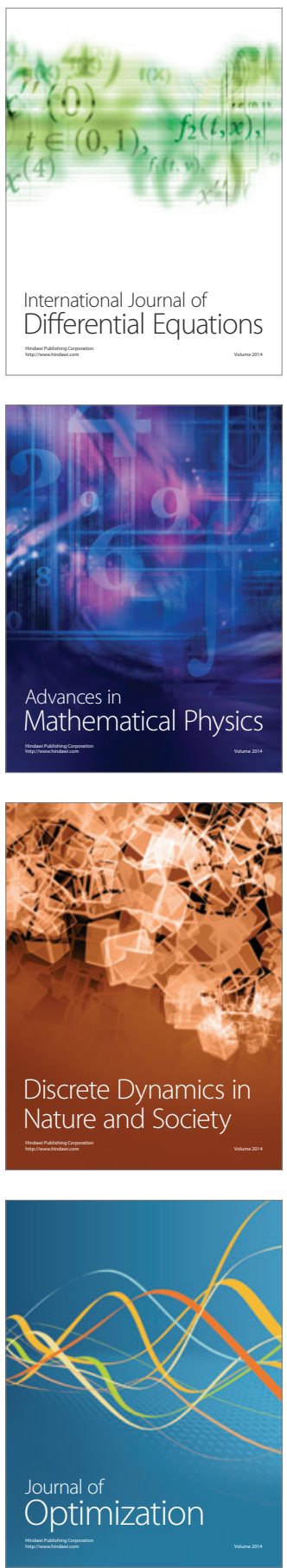